\title{
Specific Far Infrared Spectroscopic Properties of Phospholipids
}

\author{
Ruth Hielscher ${ }^{1,2}$ and Petra Hellwig ${ }^{1}$ \\ ${ }^{1}$ Laboratoire de Spectroscopie Vibrationnelle et Electrochemie des Biomolécules, Institut de Chimie, \\ Université de Strasbourg, UMR 7177, 1 rue Blaise Pascal, 67000 Strasbourg Cedex, France \\ ${ }^{2}$ Strahlentherapie und Radioonkologie, Universität Göttingen, Roberto Koch Strasse 40, \\ 37075 Göttingen, Germany
}

Correspondence should be addressed to Petra Hellwig, hellwig@unistra.fr

Copyright (C) 2012 R. Hielscher and P. Hellwig. This is an open access article distributed under the Creative Commons Attribution License, which permits unrestricted use, distribution, and reproduction in any medium, provided the original work is properly cited.

\begin{abstract}
We describe the specific spectral signature of different phospholipids and sphingolipids in the far infrared. Three specific spectral domains have been found: the head group contributions $\left(600\right.$ and $\left.480 \mathrm{~cm}^{-1}\right)$; the modes of the torsion motion of the hydrocarbon chains and of the skeleton vibration (460 to $180 \mathrm{~cm}^{-1}$ ); and the hydrogen-bonding continuum (below $300 \mathrm{~cm}^{-1}$ ). Marker bands for individual phospholipids are distinguished.
\end{abstract}

Keywords: Bound water, far infrared, hydrogen bonds, self-organization, phospholipids

\section{Introduction}

In natural membranes, a specific mixture of lipids is typically found for each organism [1]. The cell membranes of eukaryotes essentially consist of three classes of lipids: phospholipids, sphingolipids, and cholesterol. Inositol phospholipids are ubiquitous membrane lipids found in mammals as well as in bacteria and other organisms, including plants [2,3]. Cardiolipin (CL) is a major mitochondrial anionic phospholipid with important functions in promoting cell growth, anaerobic metabolism, mitochondrial function, and biogenesis. The function of this unique phospholipid is based on two protonable phosphate groups [4] that have been shown to form an intermolecular hydrogen bond with its phosphate group [5]. Phosphatidylglycerol (PG) is a crucial phospholipid as it is the metabolic precursor in the biosynthetic pathway leading to the formation of cardiolipin and the sole phospholipid of thylakoid membranes of prokaryotic and eukaryotic oxygenic photosynthetic organisms. Sphingomyelin (SM) belongs to the sphingolipid group and it is at the same time the most abundant component of mammalian membranes in brain and nervous tissues. It plays an important role for apoptosis, aging, and signal transduction with cations [6]. Phosphatidylethanolamine (PE) is ubiquitous in eukaryotic cell membranes, being unevenly distributed between the inner and the outer leaflets of the bilayer. Phosphatidylcholine (PC) is usually 
dominating in animal and plants, often amounting to almost $50 \%$ of the total lipid concentration, and as such it is obviously the key building block of membrane bilayers.

This rich molecule group, its composition and interaction, was extensively studied by means of infrared spectroscopic techniques [7, 8]. Importantly, functional groups involved in hydrogen bonding have IR absorption bands that are temperature and pressure dependent [9-11]. Different specific spectral regions can be distinguished, and so-called IR continua have been described involving hydrogen bonding features [12-15]. In the far infrared (FIR) region, this continuum is seen at about 400 to $0 \mathrm{~cm}^{-1}$, the exact position and broadness depending on the polarizability of the hydrogen bonding features. Hydrogen bonds from water molecules were found to contribute to this same spectral region [14] as recently described for the reorganization of the water solvation shell in the picosecond time range in the $\mathrm{THz}$ $[16,17]$.

Recently, we corroborated the temperature dependent phase transition from phospholipids in the mid-infrared [18-20] with the hydrogen bonding interaction seen in the far domain [21] and found that the lipid structure influenced the intermolecular hydrogen bonding interplay. In this work we examine in detail the specificity of the far infrared spectral range for the different types of phospholipids.

\section{Materials and Methods}

\subsection{Sample Preparation}

L- $\alpha$-Phosphatidylinositol (PI) from Glycine max (soybean), containing 50\% of pure PI (amount of phosphatidylethanolamine and phosphatidyl acid), 1,2-dimyristoyl-sn-glycero-3-phosphocholine (DMPC), and 1,2-dimyristoyl-sn-glycero-3-phosphoethanolamine (DMPE) were purchased from Sigma-Aldrich. Asolectin from soybean which contains equal proportions of PC, PE, and PI was purchased from Sigma-Aldrich. Phosphatidylcholine from egg yolk (EPC), phosphatidylethanolamine from egg yolk (EPE), phosphatidylglycerol from egg yolk (EPG), and sphingomyelin from egg yolk (ESM) were purchased from Lipoid (Ludwigshafen, Germany). The purity of all phospholipids is above $98 \%$ and phospholipids were used without further purifications. The samples were dissolved in chloroform using a concentration of $20 \mathrm{mg} / \mathrm{mL}$. For the lipid titrations, EPC and EPE were dissolved in a ratio of $1: 1,2: 1$ and $4: 1(\mathrm{w} / \mathrm{v})$. Furthermore, PC, PE, and PI as well as EPC, EPE, and PI were added in a ratio of $1: 1: 1(\mathrm{w} / \mathrm{v})$.

\subsection{Spectroscopy}

Far infrared absorbance spectra were recorded from 700 to $50 \mathrm{~cm}^{-1}$ with a diamond ATR unit at room temperature. Mid-infrared absorbance spectra were recorded from 4000 to $900 \mathrm{~cm}^{-1}$ with a silicon/ZnSe ATR unit. The lipid solutions were dried on the respective ATR untit. After drying, the film was rehydrated by adding $1 \mu \mathrm{L}$ distilled water to the film.

Data was recorded from 700 to $50 \mathrm{~cm}^{-1}$ with a Bruker Vertex FTIR spectrometer equipped with a silicon beamsplitter as well as a deuterated triglycine sulfate detector and a conventional infrared light source. Five spectra with 128 scans were averaged. The resolution of all spectra was $4 \mathrm{~cm}^{-1}$ and the data were acquired in the single-side, forward-backward mode. The resultant interferograms were apodized with the Blackman-Harris-3-term function and Fourier transformed with a zero filling factor of two. 


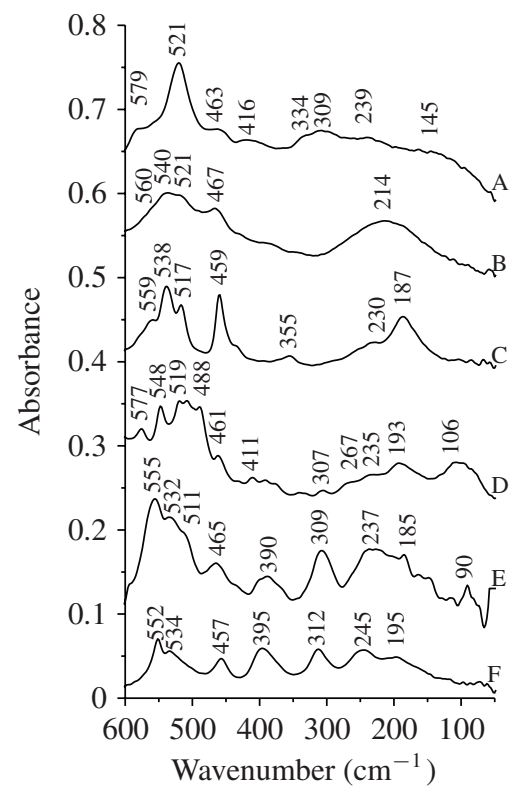

Figure 1: Far infrared absorbance spectra of different phospholipids from 600 to $50 \mathrm{~cm}^{-1}$ measured at room temperature: (A) phosphatidylinositol from Glycine max, (B) cardiolipin from bovine heart, (C) phosphatidylglycerol from egg yolk, (D) sphingomyelin from egg yolk, (E) phosphatidylethanolamine (DMPE), and (F) phosphatidylethanolamine from egg yolk.

\section{Results and Discussion}

Figure 1 summarizes the far infrared absorbance spectra of selected phospholipids and sphingolipids, all of them having a special function in membranes, as mentioned in the introduction.

Trace A shows the spectra of phosphatidylinositol (PI), trace B of cardiolipin, and trace C for phosphatidyl-glycerol form egg yolk (EPG). Trace D displays the spectroscopic properties of sphingomyelin from egg yolk (ESM), trace F represents phosphatidyl-ethanolamine from egg yolk (EPE), and the direct comparison to the synthesized phosphatidylethanolamine (DMPE) is shown in trace $\mathrm{E}$. The main difference between the two samples in trace $\mathrm{E}$ and $\mathrm{F}$ is the degree of saturation of the hydrocarbon chains.

Figure 2 shows the spectroscopic properties of phosphatidylcholine from egg yolk (EPC in trace $\mathrm{A}$ ), phosphatidylcholine $\mathrm{C}^{13}$ isotopically labeled (trace $\mathrm{B}$ ), synthesized phosphatidylcholine (DMPC) (trace C), as well as deuterated $\mathrm{D}_{22}$-phosphatidylcholine (trace $\mathrm{D}$ ), and deuterated $\mathrm{D}_{35^{-}}$ phosphatidylcholine (trace E).

Three main domains can be depicted between 600 and $50 \mathrm{~cm}^{-1}$ in the far infrared spectra of the phospholipids and sphingolipids seen in Figures 1 and 2. The signals between 600 and $480 \mathrm{~cm}^{-1}$ originate from the head group domain. Between 540 and $503 \mathrm{~cm}^{-1}$, the $\mathrm{O}-\mathrm{P}-\mathrm{O}$ wagging and rocking vibrations from the lipid head groups can be expected $[22,23]$. In these lines the absorbance band at $577 \mathrm{~cm}^{-1}$ in ESM (Figure 1, trace D) is assigned to the $\mathrm{CN}^{+}\left(\mathrm{CH}_{3}\right)_{3}$ deformation vibration. In PC this band appears at $575 \mathrm{~cm}^{-1}$ (Figure 2). In the deuterated $\mathrm{D}_{35}-\mathrm{PC}$ variant, the related $\mathrm{CN}^{+}\left(\mathrm{CD}_{3}\right)_{3}$ deformation vibration can be found at $542 \mathrm{~cm}^{-1}$ [8]. The out-of-plane deformational motion of the 


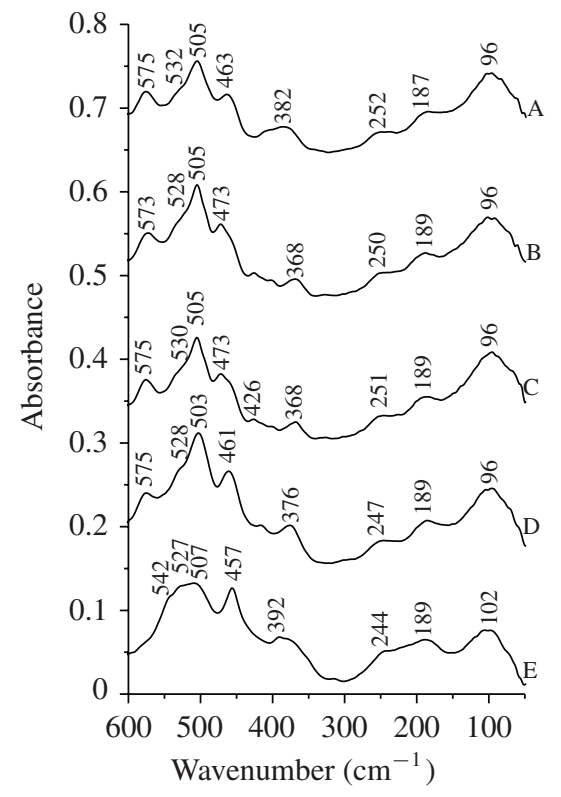

Figure 2: Far infrared absorbance spectra of different variants of phosphatidylcholine from 600 to $50 \mathrm{~cm}^{-1}$ measured at room temperature. (A) phosphatidylcholine from egg yolk, (B) phosphatidylcholine ${ }^{13} \mathrm{C}$ isotopically labeled, (C) phosphatidylcholine (DMPC), (D) deuterated $\mathrm{D}_{22}$ phosphatidylcholine, and (E) deuterated $\mathrm{D}_{35}$-phosphatidylcholine.

glycerol group in PG and CL is found about $560 \mathrm{~cm}^{-1}$. Furthermore, the deformation vibration of the ethanolamine head group of PE is seen at $555 \mathrm{~cm}^{-1}$.

A second spectral domain is defined from 460 to $180 \mathrm{~cm}^{-1}$. It includes the coordinates of the torsion motions of the hydrocarbon chains as well as the skeleton vibration of the lipid molecules. The $\mathrm{C}-\mathrm{CN}$ torsion vibration of the choline group is found around $382 \mathrm{~cm}^{-1}$. The vibration of the hydrocarbon chains has its maximum at about $185 \mathrm{~cm}^{-1}$ and it is not affected by deuteration. The $\mathrm{CH}_{3}$ torsion mode has its maximum at approximately 250 to $230 \mathrm{~cm}^{-1}$, the exact position depending on the lipid type.

The third prominent spectral region that can be depicted is the broad feature between 170 and $50 \mathrm{~cm}^{-1}$. This signal originates from the molecular breathing and from the intermolecular hydrogen bonding structure within the phospholipid bilayer [21, 24, 25]. Both CL and PG show a broad hydrogen bonding feature at higher frequencies between 300 and $100 \mathrm{~cm}^{-1}$. This observation is in agreement with the presence of hydrogen bonds with large proton polarizability [26]. For example in the PG bilayer, there is room for intermolecular hydrogen bonding between the glycerol hydroxyl groups and the phosphate moiety of the polar head group [27-30]. The hydroxyl groups in the head groups may also partially mimic the solvation properties of water. The hydrogen bonding feature of PC variants found from 165 to $50 \mathrm{~cm}^{-1}$ is just slightly affected by the deuteration of the chains. This is not surprising since isotope labeling does not strongly affect these vibrations. While deuteration is widely used in the mid-IR to perform assignments, in the far IR, deuteration is not very efficient, since the shift of the hydrogen bond is about $1-5 \mathrm{~cm}^{-1}[14,31]$. 


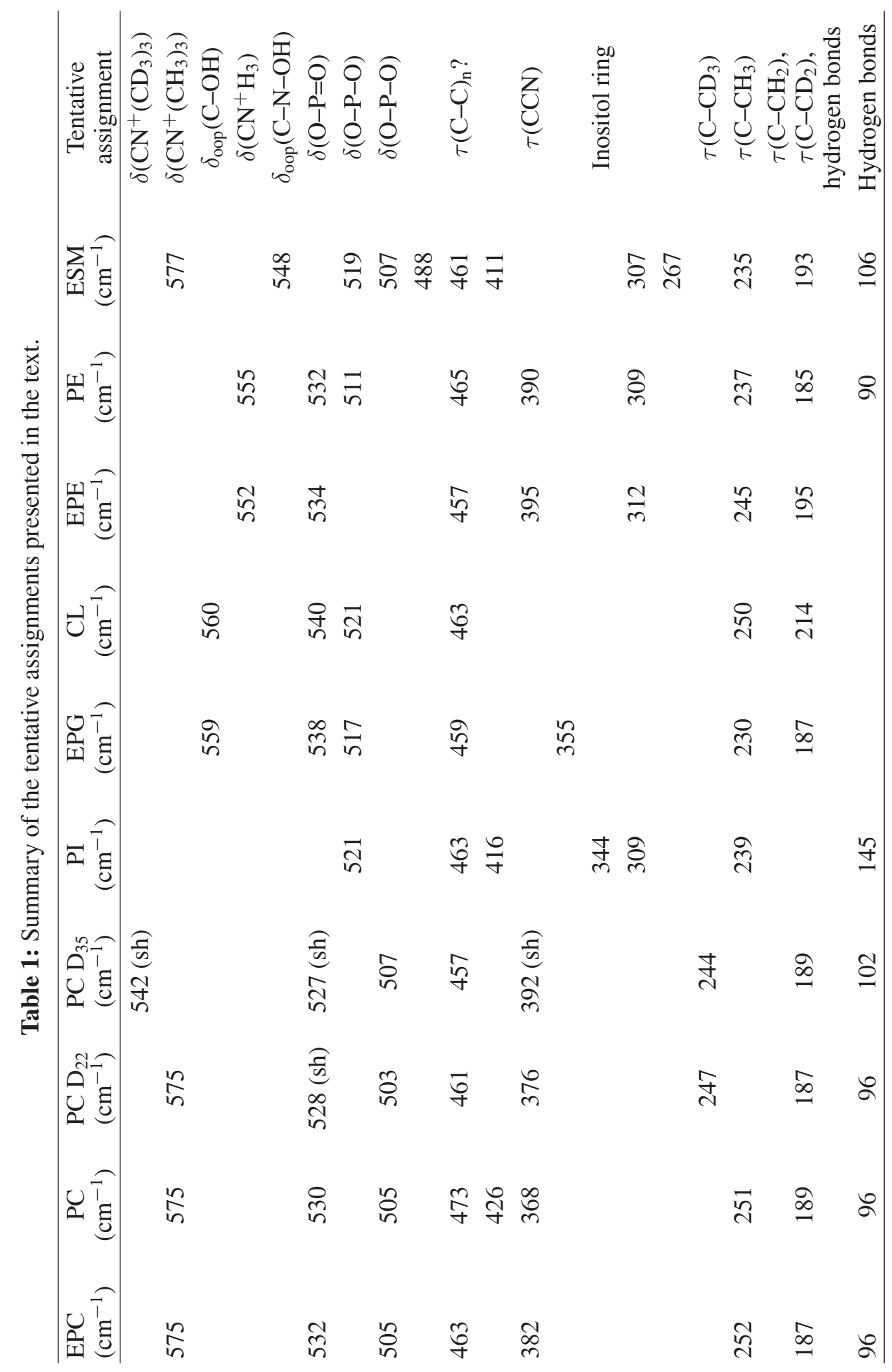


Table 1 summarizes the tentative assignments for the phospholipid contributions in the far infrared region.

\section{Conclusion}

Herein, we have presented the characterization of the far infrared spectra of different phospholipids and sphingolipids in the spectral range from 600 to $50 \mathrm{~cm}^{-1}$. The far infrared spectra of phospholipids have been divided in three main domains and in addition the individual fingerprint of each lipid was depicted. The first domain included the significant signals between 600 and $480 \mathrm{~cm}^{-1}$ which originates from the head group domain. The second domain described the range from 460 to $180 \mathrm{~cm}^{-1}$. It included the vibrations of the torsion motions of the hydrocarbon chains as well as the skeleton vibration of lipids. Finally, the third domain discussed below $300 \mathrm{~cm}^{-1}$ was assigned to the broad hydrogen bonding signature which originates from the molecular breathing and from the intermolecular hydrogen bonding structure within the lipid layer.

In conclusion, the far infrared spectral range allows the description specific signals for lipids.

\section{Acknowledgments}

This work was supported by the Centre National de la Recherche Scientifique (CNRS) and the Université de Strasbourg. The authors are grateful to Agence Nationale de la Recherche (ANR), Chaire d'excellence, for financial support. They are indebted to Drs. Regine Süss and Martin Holzer for lipid samples and discussions.

\section{References}

[1] K. M. Merz and B. Roux, Biological Membranes A Molecular Perspective from Computation and Experiment, Birkhäuser, Boston, Mass, USA, 1996.

[2] K. J. Einspahr and G. A. Thompson, "Transmembrane signaling via phosphatidylinositol 4,5bisphosphate hydrolysis in plants," Plant Physiology, vol. 93, no. 2, pp. 361-366, 1990.

[3] M. G. Low, "Biochemistry of the glycosyl-phosphatidylinositol membrane protein anchors," Biochemical Journal, vol. 244, no. 1, pp. 1-13, 1987.

[4] T. H. Haines and N. A. Dencher, "Cardiolipin: a proton trap for oxidative phosphorylation," FEBS Letters, vol. 528, no. 1-3, pp. 35-39, 2002.

[5] W. Hübner, H. H. Mantsch, and M. Kates, "Intramolecular hydrogen bonding in cardiolipin," Biochimica et Biophysica Acta, vol. 1066, no. 2, pp. 166-174, 1991.

[6] K. Sugimori, H. Kawabe, H. Nagao, and K. Nishikawa, "A DFT study of infrared spectrum of sphingomyelin lipid molecule with calcium cation," International Journal of Quantum Chemistry, vol. 108, no. 15, pp. 2935-2942, 2008.

[7] F. R. Van De Voort, J. Sedman, and T. Russin, "Lipid analysis by vibrational spectroscopy," European Journal of Lipid Science and Technology, vol. 103, no. 12, pp. 815-826, 2001.

[8] R. N. A. H. Lewis and R. N. McElhaney, "Fourier transform infrared spectroscopy in the study of hydrated lipids and lipid bilayer membranes ," in Infrared Spectroscopy of Biomolecules, H. H. Mantsch and D. Chapman, Eds., pp. 159-202, Wiley-Liss, New York, NY, USA, 1996. 
[9] R. C. Dougherty, "Temperature and pressure dependence of hydrogen bond strength: a perturbation molecular orbital approach," Journal of Chemical Physics, vol. 109, no. 17, pp. 7372-7378, 1998.

[10] G. A. Jeffery, An Introduction to Hydrogen Bonding, Oxford Press, New York, NY, USA, 1997.

[11] G. C. Pimental and A. M. MacClellan, The Hydrogen Bond, Freeman, San Francisco, Calif, USA, 1960.

[12] G. Zundel, B. Brzezinski, and J. Olejnik, "On hydrogen and deuterium bonds as well as on $\mathrm{Li}^{+}$, $\mathrm{Na}^{+}$and $\mathrm{Be}^{2+}$ bonds: IR continua and cation polarizabilities," Journal of Molecular Structure, vol. 300, pp. 573-592, 1993.

[13] F. Garczarek and K. Gerwert, "Functional waters in intraprotein proton transfer monitored by FTIR difference spectroscopy," Nature, vol. 439, no. 7072, pp. 109-112, 2006.

[14] J. B. Brubach, A. Mermet, A. Filabozzi, A. Gerschel, and P. Roy, "Signatures of the hydrogen bonding in the infrared bands of water," Journal of Chemical Physics, vol. 122, no. 18, Article ID 184509, pp. 1-7, 2005.

[15] R. Hielscher, T. Friedrich, and P. Hellwig, "Far- and mid-infrared spectroscopic analysis of the substrate-induced structural dynamics of respiratory complex i," ChemPhysChem, vol. 12, no. 1, pp. 217-224, 2011.

[16] U. Heugen, G. Schwaab, E. Bründermann et al., "Solute-induced retardation of water dynamics probed directly by terahertz spectroscopy," Proceedings of the National Academy of Sciences of the United States of America, vol. 103, no. 33, pp. 12301-12306, 2006.

[17] S. Ebbinghaus, J. K. Seung, M. Heyden et al., "An extended dynamical hydration shell around proteins," Proceedings of the National Academy of Sciences of the United States of America, vol. 104, no. 52, pp. 20749-20752, 2007.

[18] H. H. Mantsch, A. Martin, and D. G. Cameron, "Characterization by infrared spectroscopy of the bilayer to nonbilayer phase transition of phosphatidylethanolamines," Biochemistry, vol. 20, no. 11, pp. 3138-3145, 1981.

[19] A. V. Popova and D. K. Hincha, "Intermolecular interactions in dry and rehydrated pure and mixed bilayers of phosphatidylcholine and digalactosyldiacylglycerol: a fourier transform infrared spectroscopy study," Biophysical Journal, vol. 85, no. 3, pp. 1682-1690, 2003.

[20] H. Binder, "Water near lipid membranes as seen by infrared spectroscopy," European Biophysics Journal, vol. 36, no. 4-5, pp. 265-279, 2007.

[21] R. Hielscher and P. Hellwig, "The temperature-dependent hydrogen-bonding signature of lipids monitored in the far-infrared domain," ChemPhysChem, vol. 11, no. 2, pp. 435-441, 2010.

[22] K. Leberle, I. Kempf, and G. Zundel, "An intramolecular hydrogen bond with large proton polarizability within the head group of phosphatidylserine. An infrared investigation," Biophysical Journal, vol. 55, no. 4, pp. 637-648, 1989.

[23] M. Klähn, G. Mathias, C. Kötting et al., "IR spectra of phosphate ions in aqueous solution: predictions of a DFT/MM approach compared with observations," Journal of Physical Chemistry A, vol. 108, no. 29, pp. 6186-6194, 2004.

[24] E. Knözinger, "Far-infrared fourier spectroscopy as a method for structure determination in chemistry," Angewandte Chemie International Edition, vol. 15, no. 1, pp. 25-39, 1976.

[25] E. Knözinger, "Ferninfrarot-Fourier-Spektroskopie als Methode zur Strukturaufklärung in der Chemie," Angewandte Chemie, vol. 88, no. 1, pp. 1-16, 1976.

[26] G. Zundel, "The far infrared vibration of hydrogen bonds with large proton polarizability," Journal of Molecular Structure, vol. 381, no. 1-3, pp. 23-37, 1996.

[27] J. M. Boggs, "Intermolecular hydrogen bonding between lipids: influence on organization and function of lipids in membranes," Canadian Journal of Biochemistry, vol. 58, no. 10, pp. 755$770,1980$. 
[28] J. M. Boggs, "Effect of lipid structural modifications on their intermolecular hydrogen bonding interactions and membrane functions," Biochemistry and Cell Biology, vol. 64, no. 1, pp. 50-57, 1986.

[29] J. M. Boggs, "Lipid intermolecular hydrogen bonding: influence on structural organization and membrane function," Biochimica et Biophysica Acta, vol. 906, no. 3, pp. 353-404, 1987.

[30] I. Pascher, S. Sundell, K. Harlos, and H. Eibl, "Conformation and packing properties of membrane lipids: the crystal structure of sodium dimyristoylphosphatidylglycerol," Biochimica et Biophysica Acta, vol. 896, no. 1, pp. 77-88, 1987.

[31] J. R. Powell, F. M. Wasacz, and R. J. Jakobsen, "Algorithm for the reproducible spectral subtraction of water from the FT-IR spectra of proteins in dilute solutions and adsorbed monolayers," Applied Spectroscopy, vol. 40, no. 3, pp. 339-344, 1986. 


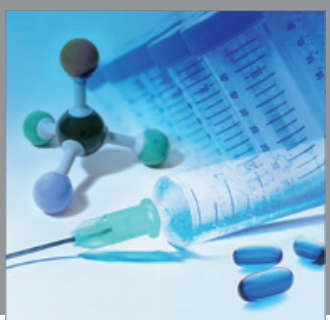

International Journal of

Medicinal Chemistry

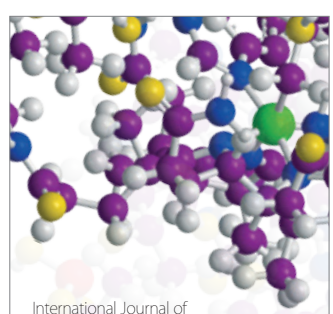

Carbohydrate Chemistry

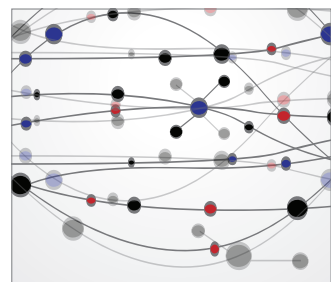

The Scientific World Journal
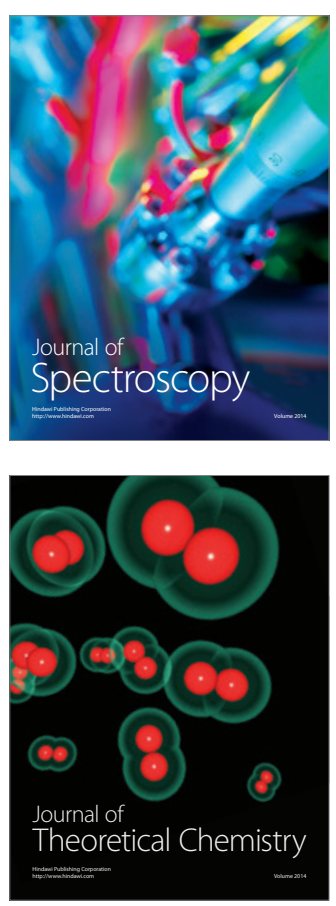
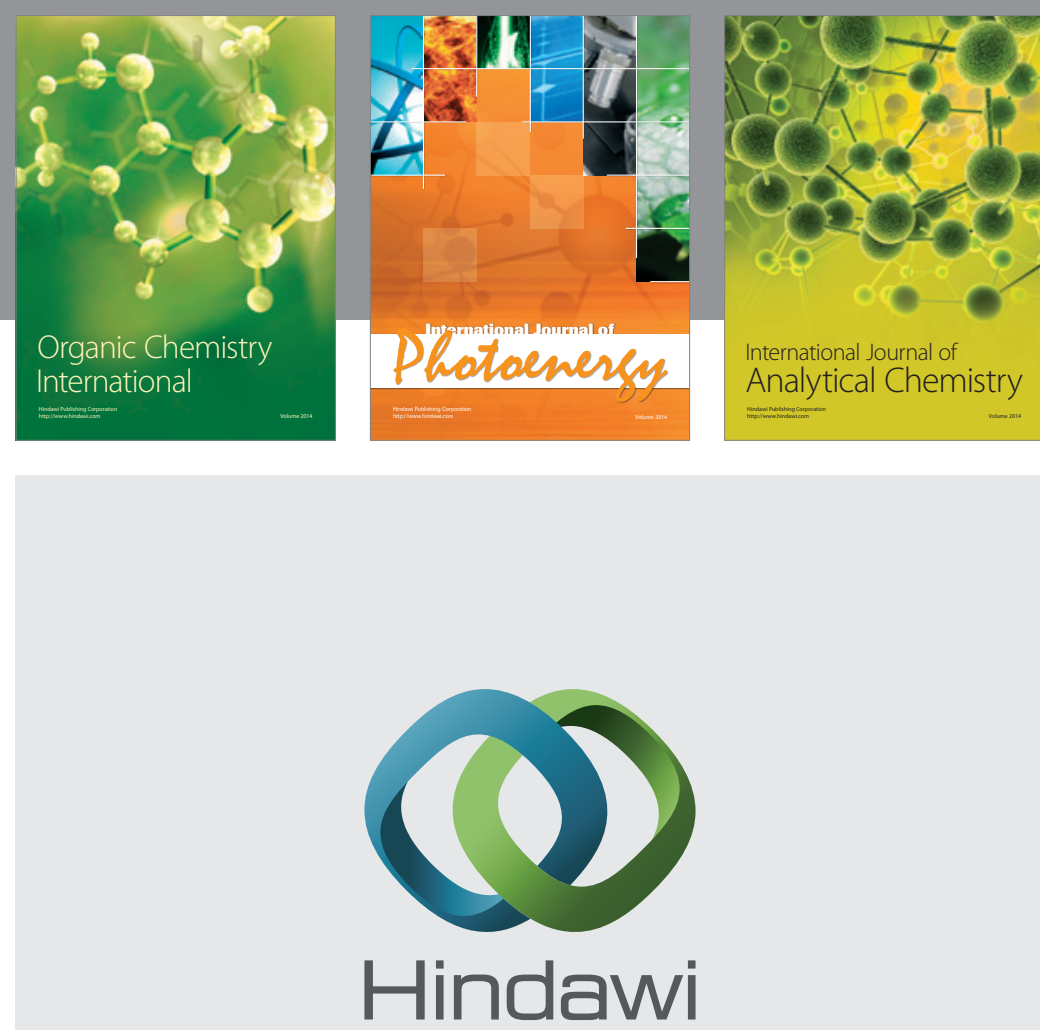

Submit your manuscripts at

http://www.hindawi.com
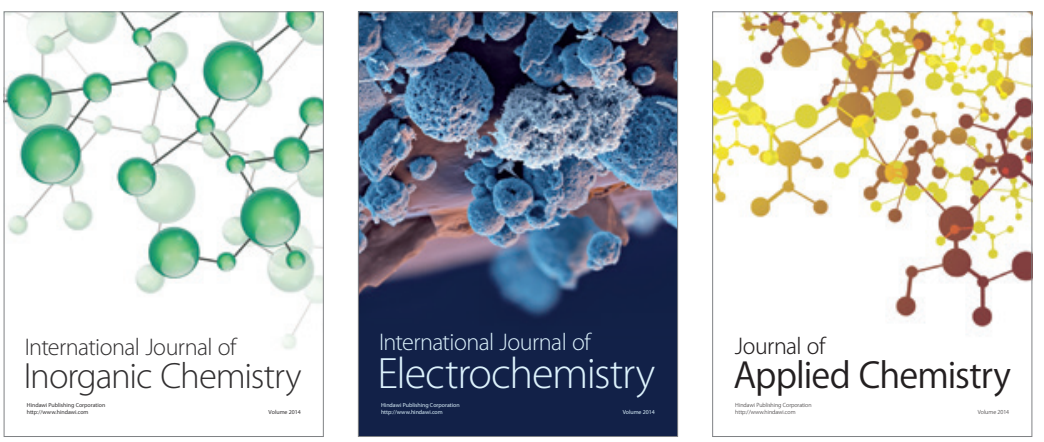

Journal of

Applied Chemistry
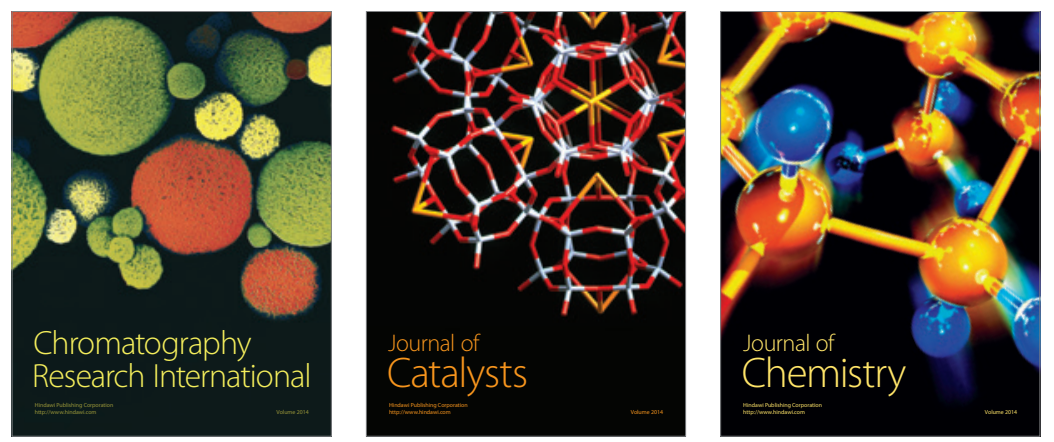
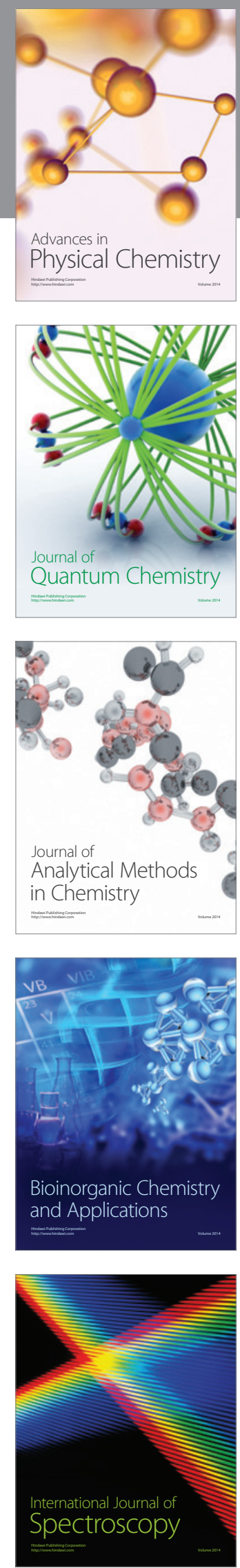